\title{
Bias in Clinical Practice
}

\author{
Satish V. Khadilkar ${ }^{1}$. Suvarna S. Khadilkar ${ }^{2}$
}

Received: 30 December 2019 / Accepted: 30 December 2019 / Published online: 16 January 2020

(C) Federation of Obstetric \& Gynecological Societies of India 2020

\begin{abstract}
Role of bias in errors of decision making is receiving increasing attention. It is turning out to be one of the main sources of mistakes. Hence, it is important to be aware of biases and to design strategies toward an unbiased approach. Biases are of various types, and the potential sources of bias can be related to the consultant, patients and factors related to working conditions. Availability bias, base rate neglect, confirmation bias, conjunction rule, diagnostic momentum bias, framing effect and confirmation bias are the common types, and these have been discussed in this manuscript using a scenario-based format. Two types of human thinking, the rapid intuitive mode and the slow reflective mode, their pros and cons and their role in biases are discussed. Strategies to enhance awareness of biases, tips to improve reasoning, promote freethinking, enhance decision-making skills and resorting to checklists have been deliberated to achieve an unbiased approach.
\end{abstract}

Keywords Bias in practice $\cdot$ Conjunction rate $\cdot$ Availability bias $\cdot$ Diagnostic momentum $\cdot$ Framing effect $\cdot$ Confirmation bias $\cdot$ Base rate neglect $\cdot$ Commission bias

'The unexamined thought is not worth thinking'- Socrates.

\section{Introduction}

Bias in clinical medicine is an extremely important and under recognized area. It is believed that diagnostic errors are associated with $6-17 \%$ of adverse events in hospitals and $28 \%$ of these are attributed to cognitive errors [1]. Cognitive bias accounts for $70 \%$ of diagnostic errors, and knowledge deficit contributes to a very minute proportion [2].

Dr. Satish V. Khadilkar MD, DM, DNBE, FIAN, FICP, FAMS, FRCP (London) is presently the dean and professor and head at the Department of Neurology at the Bombay Hospital Institute of Medical Sciences, Mumbai. Prof. Suvarna Satish Khadilkar MD DGO FICOG, CIMP, Diploma in Endocrinology (UK), is editorin-chief of Journal of Obstetrics and Gynecology of India and Treasurer, FOGSI.

Suvarna S. Khadilkar

suvarnakhadilkar@yahoo.com;

suvarnakhadilkar2@gmail.com

1 110, New Wing, First Floor, Bombay Hospital, 12, New Marine Lines, Mumbai, India

2 Bombay Hospital and Medical Research Centre, 12, New Marine Line, Mumbai 400020, India
Medical interactions, such as consultations and procedures, take place between two individuals, and hence each one is unique and open to bias. As shown in Table 1, potential sources of bias are consultant related or patient related. Some others can be situational and circumstantial. Clearly, every day and every decision are different, subject to various factors. For example, if you have had a surgical mishap, when you take the next patient up, the previous case will linger on in your mind and may affect your judgment!

While unbiased decision making is based on intelligence, experience, and objective assessment, in reality, biases and prejudices often complicate and influence decision making. Bias arises from beliefs, and beliefs are linked to the scientific, cultural and social inputs received by the individual over his or her life and need not be accurate; moreover curiously, it is often independent of intelligence [3].

This manuscript will look at various biases which one may encounter in clinical practice as obstetrician and gynecologist.

\section{Scenario 1}

A practitioner attends a mortality meeting where death of a patient due to massive intra-abdominal hemorrhage from a misdiagnosed ectopic pregnancy is presented. We were 
Table 1 Potential sources of bias

Consultant related
Overwork and mental fatigue
Availability of confounding information
Emotional involvement with the patients
Clouding judgment of the consultant
Patient related
Multiple and complex medical or surgical ailments
Inability to express symptoms, interaction issues
Others
Inadequacy of time, information, lack of available paradigms, etc.

taught "To Think Ectopic" in older times. But in today's day and age, deaths due to ectopic pregnancy are very rare, because of easy accessibility and availability of ultrasonography. The doctor returns to the consulting rooms and examines a young unmarried girl having pain in the left iliac region. An ultrasound is available and is reported to be within normal limits, and serum beta HCG test is negative. Doctor sends this patient for a CT scan to make sure that there is no ectopic pregnancy.

This is an example of the availability bias. What the mind has seen recently is recalled easily and firmly, often with undue importance. In this case, the chance of ectopic pregnancy was very little but still the patient was subjected to CT scan exposing her to radiation. This scenario teaches us, "to think Ectopic....Unbiased." There is another bias in this example: the base rate neglect! Base rate is the incidence of the given condition. Ectopic pregnancy in an unmarried girl with normal ultrasound and negative beta HCG is exceptional though not unknown. This was not kept in mind by the doctor. The base rate neglect in a given situation leads to over-investigations. Further, if investigations have falsepositive or false-negative results, it compounds the issue.

Another common example of availability bias is the oftnoted transient increase in unscheduled cesarean deliveries following the occurrence of catastrophic cases of uterine rupture or neonatal hypoxic ischemic encephalopathy in the obstetric wards.

\section{Scenario 2}

A middle-aged woman presented with general feeling of tiredness and fatigue. Her routine investigations showed leukocytosis of $11,000 / \mathrm{mm}^{3}$. The practitioner assumes that she has an infection and prescribes antibiotics.

This is the confirmation bias wherein the doctor presumes that there is an infection and seeks support of his presumption from the elevated leukocyte count. This is the situation of fitting the results in preconceived notions, rather than the reverse. An unbiased analysis would be "there is leukocytosis and I need to find the cause for it." In the words of Arthur Conan Doyle, "the temptation to form premature theories on insufficient data is the bane of our profession." We need to remember to get data before the theory and not to put data to suit our theory!

\section{Scenario 3}

A patient is admitted for eclampsia and is being treated with magnesium sulfate as anticonvulsant. During the course in ICU, she develops complete anuria. Interpreting this as acute renal failure due to eclampsia, a battery of investigations is done to prepare her for dialysis. It shows normal urea and creatinine levels. Re-evaluation uncovers a blocked catheter, which is completely unrelated to eclampsia!

This is the conjunction rule. A single unifying explanation is statistically more probable than many unrelated events occurring in the same patient, the Occam's razor. While this is generally true, Occam's razor is a broad principle and situations do exist where multiple unrelated events can be responsible for what is happening to the patient. Hence, as we discover plethora of parameters and investigative tools, it is possible that Occam's razor will need to be put to rigorous testing.

\section{Scenario 4}

Senior doctor makes a plan for the cesarean section of a primigravida with breech presentation in antenatal outpatient department. The patient, however, gets admitted with strong labor pains in emergency. The junior doctor on duty immediately plans to prepare her for cesarean section on seeing the advice of the senior doctor on antenatal clinic card, ignoring the fact that in the meantime patient is already fully dilated. He does not change the plan as the earlier plan was made by the senior colleague, so patient is shifted to the operation theater and patient delivers on the trolley.

This is the diagnostic momentum bias. So often on the ward rounds, a plan made by a senior and respected member of the fraternity remains operational without the doctor on-site acting on the ongoing changes in the patient's condition. When asked about it, the younger doctor justifies that "management plan was made by a more senior colleague."

\section{Scenario 5}

A new drug is presented to the doctor as the most effective medicine till date. Studies from reputed journals depicting success rates of around $80 \%$ are shown: a huge positive. Other molecules depict failure rates of $10-12 \%$ !! The doctor 
prefers to use the new drug, not paying attention to the fact that $12 \%$ failure means $88 \%$ success rates!!

This bias is called the framing effect. The way the information is put up confuses the mind into believing what is presented. Most medical practitioners are not well versed with medical statistics and find it difficult to interpret studies in the correct light. While a rigorous peer-review process is expected to eliminate this bias, at times, a "statistical truth" may be presented to the common medical practitioner, who may take it at face value.

\section{Scenario 6}

A patient hears a story from her friend that she was advised hysterectomy for abnormal uterine bleeding, which she refused and later she developed cancer. The patient gets scared and consults a doctor seeking a hysterectomy. Doctor complies with her request!

This would be the commission bias. Human mind leans toward activity than inertia. Hence, acts of commission are more often seen than those of omission. Truthfully, both have negative repercussions.

\section{What is the Explanation for Biased Thinking?}

In India, where work load is a major issue, doctors are always looking for time-efficient paradigms. Hence, they resort to the oft-promoted method of pattern recognition.

Pattern recognition is a method by which you identify a set of symptoms or signs which represent a common disease condition. Pattern recognition is based on the rapid mental processing, taking cues from memory stores and intuition, and is a low energy-consuming method. While effective for the routine cases, it basically looks at the broad diagnostic label, ignoring nuances and the oddities. Moreover, the deep-rooted need for achieving a diagnosis prompts "pigeon holing," which can take precedence over facts, and ill-fitting patterns also get slotted into diagnostic categories. Thus, the advantage of this method of medicine is time efficiency at the cost of bias and missing the unusual.

The second technique is of being methodical and thoughtful, at every stage of the diagnostic process and therapy options. As some teachers have stressed, take history in entirety and keep it aside. Do a complete clinical examination, not to just confirm the diagnosis offered by history but to check things on their own merit. Then, sit down with these two independent parts of assessment and correlate them. Then, decide on which investigations you really need and how will your actions change if they are positive or negative. Based on all this information, take a decision about which procedure you will want to perform on the patient, for maximum benefit. This is a time-consuming and laborintensive process but surely will lead to fewer misadventures and give the doctor a chance to be unbiased. This method puts a lot of stress on the cognitive skills.

It has been known that the human mind is in the rapid mode of pattern recognition for most of the time, as it is a low cognitive stress process. Mind can shift to the second mode when directed, and this is usually achieved by actively slowing down. Asking yourself a question such as "Hey, what's happening here?" will slow you down and make you go in the second, more objective mode. When there is failure to account for all the data and when red flags are seen, we should reconsider and analyze the case once again in the slow reflective mode [4]. The trick perhaps lies in the understanding as to when to employ which method, for best balance of efficiency and accuracy which some astute clinicians possess.

In the expert practice model [5], two types of experts have been identified; the routine expert and the adaptive expert. Routine expert appropriately uses preexisting knowledge to quickly solve routine, familiar or uncomplicated problems (the rapid mode). The adaptive expert is able to employ a deep conceptual understanding and engage in reflection to create novel solutions for complicated or unfamiliar problems (the slow mode). Adaptive expertise builds with time, a function of having participated in multiple problem-solving exercises. Yee et al found that obstetricians who scored higher on reflective capacity tests had higher rates of successful attempts of vaginal birth after cesarean section [5].

\section{What can be Done to Develop the Ability to Take Unbiased Decisions?}

While the first step in this direction would appear to be informing doctors of the various types of bias that exist and to suggest strategies to correct these, but there is currently little evidence that it works. Also, there are very interesting studies, which show that the doctors who believe that they are unbiased in their decision making do not do well on tests given to them! So the issues are of non-recognition of one's own faults and not having very satisfactory teaching modules to rectify them.

Actively slowing down has been shown to eliminate some of the biases, and it is easy to do [6]. Deliberating on the problem can make us think in a more unbiased way. As we start thinking about a clinical problem, our mind originally directs us in a certain way, toward a certain diagnosis and the management approach. This initial process is often "intuitive" and incorporates bias. Curiously, once these thoughts are formed, intelligence does its best to defend the original thoughts; however, irrational they may be! While the mind and intelligence are busy proving 
Table 2 Steps to avoid bias

\author{
Increasing awareness of biases and providing facts \\ Departmental meetings \\ Mortality meetings \\ Case reviews with lessons \\ Sharing personal experience of complications \\ Helping improve reasoning, promote freethinking and enhance decision-making skills \\ Awareness and understanding of one's own thought process (Meta cognition sessions) \\ Counterintuitive thinking "can this be explained in any other way?" \\ Developing systematic reasoning for accurate analysis \\ Simulated case scenarios for gaining experience \\ Response from peers about decisions taken \\ Making working conditions more conducive \\ Displaying protocols and checklists \\ Availability of colleagues to discuss decisions \\ Work hour regulations and avoiding fatigue
}

the initial thoughts, it may be a good idea for doctors to ask a counterintuitive question to themselves, e.g., "Why could it not be Y, why am thinking only of X," to free their thinking. Another question to oneself "how confident am I of this diagnosis?" can be used to begin thinking of alternatives. In the words of Sherlock Holmes, "When you have eliminated the impossible, what remains, however improbable, must be the truth!"

Checklists are often recommended in clinical practice as they are known to result in a certain degree of debiasing. As the doctor goes through the checklist, he is forced to stop, think and act rationally at each point, which he may not do in his fast mode intuitive thinking. As an obstetrician, a fast mode of action is required in many life-threatening emergency situations such as peripartum hemorrhage. Display of checklists and protocols in labor room will help in taking action rapidly with accuracy in such situations [7].

Checklists are easy to create and personalize and hence a practical aid to de-biasing. These strategies are summarized in Table 2.

\section{Conclusions}

Bias pervades daily professional life of medical practitioners and affects outcomes. Being aware of various biases that exist and understanding why they occur is important in working toward unbiased decision making and execution. Rapid mental processing works on intuitive elements and does not take into account counterarguments. Slowing down, asking counterintuitive questions, delinking intelligence from beliefs and generating checklists can help the decision-making process to be unbiased and objective.

\section{References}

1. Balogh EP, Miller BT, Ball JR. Improving diagnosis in health care. Washington: National Academic Press; 2015.

2. Saber Tehrani AS, Lee H, Mathews SC, Shore A, Makary MA, Pronovost PJ, Newman-Toker DE. Twenty five-year summary of US malpractice claims for diagnostic errors 1986-2010: an analysis from the National Practitioner Data Bank. BMJ Qual Saf. 2013;22(8):672-80. https://doi.org/10.1136/bmjqs-2012001550 (Epub 2013 Apr 22).

3. Royce CS, Hayes MM, Schwartzstein RM. Teaching critical thinking: a case for instruction in cognitive biases to reduce diagnostic errors and improve patient safety. Acad Med. 2019;94(2):187-94.

4. O'sulivan ED, Schofield SJ. Cognitive bias in clinical medicine. J R Coll Phys Edinb. 2018;48:225-32.

5. Mylopoulous M, Regeher G. Putting the expert together again. Med Educ. 2011;45:920-6.

6. Dobbler CC, Morrow AS, KAmath CC. Clinician's cognitive biases: a potential barrier to the implementation of evidence based clinical practice. BMJ Evid Based Med. 2019;24(4):13740. https://doi.org/10.1136/bmjebm-2018-111074.

7. Khadilkar SS, Sood A, Ahire P. Quantification of peri-partum blood loss: training module and clot conversion factor. J Obstet Gynecol India. 2016;66:307-14. https://doi.org/10.1007/s1322 4-016-0888-9.

Publisher's Note Springer Nature remains neutral with regard to jurisdictional claims in published maps and institutional affiliations. 


\section{About the Author}

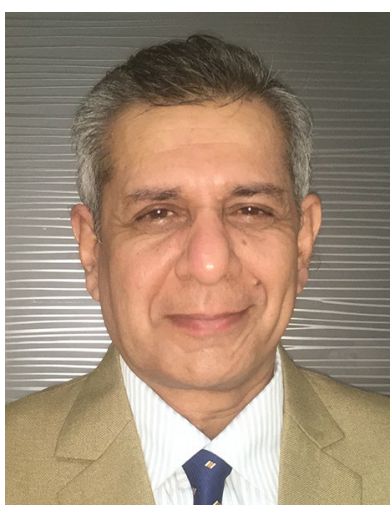

Dr. Satish V. Khadilkar is presently the dean and professor and head at the Department of Neurology at the Bombay Hospital Institute of Medical Sciences, Mumbai. $\mathrm{He}$ is the immediate past president of Indian Academy of Neurology and the past president of Maharashtra Association of Neurology and Bombay Neurological Association. He was previously editor-in chief of the Annals of Indian Academy of Neurology. He is the Fellow of
Royal College of Physicians (London), National Academy of Medical Sciences, Indian Academy of Neurology and Indian College of Physicians. He has to his credit six books, 43 book chapters and over 150 publications in national and international peer-reviewed journals. 